Lawrie, S. M., Scott, A. I. F. \& Sharpe, M. C. (200I) Implementing evidence-based psychiatry: whose responsibility? British Journal of Psychiatry, 178, 195-196.

G. Ikkos Postgraduate Centre, Edgware Community Hospital, Edgware Middlesex HA8 OAD, UK

\section{Methodological rigour in cross-cultural research}

The paper by Selten et al (2001) is of interest in the context of methodological developments in cross-cultural research which now demand a much greater level of rigour in defining racial ethnic or cultural groups to reflect the hypotheses and the interpretation of the data (McKenzie et al, 1996). One of the difficulties of international work is that each country tends to use particular labels for particular ethnic and cultural groups which reflects a country's particular experience of migration and immigration. Specifically, the term Hindustanis as used by Selten et al is not meaningful. Hindustanis refers to people of Hindu origin, a religious category. This group is compared with Turkish and Moroccans - national categories reflecting place of birth. Alternatively, Hindustanis might refer to people of Indian origin, whom Selten et al and perhaps other researchers in The Netherlands, generally might refer to as Hindustanis. To justify the use of the term Hindustanis they suggest that British Indians migrated to The Netherlands in the 19th century. Selten et al are suggesting that people retain all the risk factors and cultural beliefs that determine illness behaviour despite exposure to new cultures and that these patterns persist over generations. This is simply not the case. It would have been of more interest to explore specific social factors that might account for their findings while taking account of the specific experiences of the cultural and sub-cultural groups that were more uniformly identified. For example, the ethnic density of each of their groups in comparison to the host population may explain the rates of psychosis (Bhugra \& Jones, 2001). Social factors such as cultural identity, unemployment and separation from parents may also explain variations in rates (Bhugra et al, 1997). These factors are entirely unexplored. The use of aggregated ethnic, religious or national groups that do not relate to similar groups makes any explanation or interpretation of their findings impossible, so reinforcing the view that research on ethnic groups bears no relationship to improving service delivery or understanding distress.

Bhugra, D., Leff, J., Mallett, R., et al (1997) Incidence and outcome of schizophrenia in Whites, AfricanCaribbeans and Asians in London. Psychological Medicine, 27, 791-798.

_ \& Jones, P. (200I) Migration and mental illness. Advances in Psychiatric Treatment, 7, 208-222.

McKenzie, K., Crowcroft, N. S., De Bono, D., et al (1996) Describing race, ethnicity, and culture in medical research. BM], 312, 1054-1060.

Selten, J. P., Veen, N., Feller, W., et al (200I) Incidence of psychotic disorders in immigrant groups to The Netherlands. British Journal of Psychiatry, 178, 367-372.

K. Bhui St Bartholomew's \& The Royal London School of Medicine and Dentistry, Department of Psychiatry, Basic Medical Sciences Building, Queen Mary \& Westfield College, Mile End Road, London El 4NS, UK

D. Bhugra Institute of Psychiatry, London, UK

Author's reply: Drs Bhui \& Bhugra write that categories are confused by comparing Turkish and Moroccan people with Hindustanis. However, it is perfectly clear from the text and the tables that people were classified by country of birth (or country of birth of the parents) and that immigrants from Turkey or Morocco were compared with immigrants from (the country of) Surinam.

We defined Hindustanis as people who migrated in the 19th century from British India to Surinam (not to The Netherlands, as Drs Bhui \& Bhugra suggest). In The Netherlands, the designation Hindustani is not a religious category and small proportions are Muslim or Buddhist.

Drs Bhui and Bhugra note that social factors, such as ethnic density, cultural identity, unemployment and separation from parents, are "entirely unexplored". Apparently, it escaped their attention that the proportions of children growing up in single-parent families were presented on p. 367 (Selten $e t$ al, 2001). This proportion is lowest for the group with the highest schizophrenia risk (Moroccans). Similarly, the pattern of unemployment rates (higher for Turkish and Moroccan people than for Surinamese or Antilleans) does not resemble the pattern of schizophrenia rates (lowest for Turkish people) (Selten \& Sijben, 1994). It follows that an explanation in terms of social factors is not as easy as suggested.

Drs Bhui and Bhugra correctly argue that attention should be paid to ethnic differences. Our study reported an increased incidence of schizophrenia among Surinamese citizens of The Hague, mostly Hindustanis. One might ask, therefore, whether the increased incidence also applies to African Surinamese immigrants, who have common origins with AfricanCaribbeans in the UK. An answer can perhaps be found indirectly. When they migrated to The Netherlands in the 1970s and early 1980s, Hindustanis and African Surinamese differed greatly in their preference for a Dutch residence. As a result, the ethnic composition of the Surinamese community in Amsterdam (70\% African and $26 \%$ Hindustani) is different from that in The Hague (10\% African and $80 \%$ Hindustani) (Martens \& Verweij, 1997). The Dutch psychiatric registry gave information on the residence of Surinamese-born people who had been discharged with an ICD diagnosis of schizophrenia in the period 1978-1996. The Central Bureau for Statistics provided figures for the Surinameseborn populations in Amsterdam and The Hague on 1 January 1990 (mid-period). The age structures of both populations were very similar. The results can be summarised as follows. In The Hague: 23815 (Surinamese-born) citizens in 1990; 253 citizens admitted over the 19-year period. In Amsterdam: 38722 (Surinamese-born) citizens in 1990; 493 admitted. These results although not definitive, suggest that the incidence of schizophrenia is also increased for African Surinamese immigrants. This conclusion is supported by clinical experience and a small incidence study in Amsterdam (Dekker et al, 1996).

Dekker, J., Peen, J., Heijnen, H., et al (1996) Psychiatric admissions in Amsterdam by ethnicity and diagnosis (in Dutch). Nederlands Tijdschrift voor Geneeskunde, 140, 368-371.

Martens, E. P. \& Verweij, A. O. (1997) Surinamese in the Netherlands. Rotterdam: Institute for SocioEconomic Research.

Selten, J. P. \& Sijben, N. (1994) First admission rates for schizophrenia in immigrants to the Netherlands. Social Psychiatry and Psychiatric Epidemiology, 29, 7I-77.

_, Veen, N., Feller, W., et al (200I) Incidence of psychotic disorders in immigrant groups to The Netherlands. British Journal of Psychiatry, I78, 367-372.

J. P. Selten Department of Psychiatry, University Medical Centre, PO Box 85500, 3508 GA Utrecht, The Netherlands 